\title{
Increased HIV Risk Associated with Criminal Justice Involvement among Men on Methadone
}

\author{
Matthew Epperson, Nabila El-Bassel, Louisa Gilbert, E. Roberto Orellana, and Mingway \\ Chang \\ Social Intervention Group, Columbia University School of Social Work, 1255 Amsterdam Avenue, \\ New York, NY 10027, USA \\ Matthew Epperson: mwe2102@columbia.edu
}

\begin{abstract}
This paper examines the relationship between HIV risk and criminal justice involvement among a random sample of 356 men enrolled in methadone maintenance treatment programs in New York City. Bivariate and logistic regression analyses were performed to estimate the associations between measures of criminal justice involvement and participant HIV risk, controlling for sociodemographic variables. A lifetime history of incarceration was significantly associated with being HIV positive (Adjusted OR $=5.08$ ). Recent arrest was associated with unprotected vaginal sex and having multiple female sexual partners. Sex trading was associated with both arrest and incarceration, and the strongest association was found between selling sex and recent incarceration (Adjusted OR $=5.69$ ). Results suggest that recent criminal justice involvement among men with substance abuse histories is associated with increased HIV risk behaviors. Findings underscore the need for targeted HIV prevention efforts for men on methadone with a recent history of arrest or incarceration.
\end{abstract}

\section{Keywords}

HIV risk; Men; Criminal justice; Substance abuse; Methadone

\section{Introduction}

An area of research that has gained considerable recognition over the last 10 years is the increasing prevalence of HIV and AIDS in the criminal justice system (Boutwell and Rich 2004; Braithwaite et al. 2002; Vlahov and Putnam 2006). This issue has been identified as a serious global public health concern (Seal 2005). Prevalence of HIV among U.S. prisoners has been demonstrated to be three to four times that of the general population and, since 1991, the rate of confirmed AIDS cases has consistently been higher among prison inmates than in the general population (Maruschak 2004, 2005; Rich et al. 1999). It is estimated that $25 \%$ of all HIV-infected Americans pass through the U.S. correctional system annually (Braithwaite and Arriola 2003). A study by the New York City Department of Health (1999) revealed that 9.7\% of the city's jail inmates tested positive for HIV ( $7.6 \%$ for males and $18.1 \%$ for females).

Although twice as many offenders are under community supervision as are incarcerated, most HIV research in this area has focused on incarcerated populations; few studies have examined HIV/AIDS and justice-involved individuals in the community (Glaze 2003). Belenko et al. (2004) found that $12 \%$ of 140 men on probation and parole were HIV positive, indicating that 
high HIV prevalence is not restricted to custodial settings. Although intraprison HIV transmission has been proposed as a contributing cause to high HIV prevalence (Krebs and Simmons 2002), the transmission of HIV outside of custodial settings is suggested as a much greater threat due to greater access to drugs and sexual activity (Belenko et al. 2004; Spaulding et al. 2002). Many offenders circulate between jails and the community, and offenders in the community may relapse into behaviors that increase HIV risk and raise distinct health concerns (Hammett et al. 1998; Pollack et al. 1999). Successful transition and integration from incarceration to community settings, including access to drug treatment and health care, can play an important role in reducing HIV risks for this vulnerable population. Community-based criminal justice settings present a prime opportunity for HIV prevention interventions (Freudenberg et al. 2005; Hammett 2006; Spaulding et al. 2002; Springer and Altice 2005). Understanding the context of HIV risk for community-based offenders, however, is a key factor in addressing prevention efforts with this population.

HIV risk for male offenders has been primarily explained by the increased prevalence of substance abuse in criminal justice populations and the association between substance abuse and HIV risk behaviors. Specifically, about $80 \%$ of the U.S. prison population was heavily involved in drug and alcohol use prior to incarceration (Harrison and Beck 2003; Stephens et al. 2006). It is estimated that more than 20,000 offenders with a recent history of injection drug use enter the New York City criminal justice system every year (Belenko et al. 2004). Elevated sexual HIV risk behaviors have been associated with excessive use of alcohol (Bryant 2006), marijuana and cocaine (Braithwaite and Stephens 2005), methamphetamines (Molitor et al. 1998; Wohl et al. 2002), and club drugs (Colfax and Guzman 2006). Risky sexual practices were also found to be associated with crack cocaine use and polysubstance abuse (Booth et al. 1993; Campsmith et al. 2000; Chesney et al. 1998; Stephens et al. 2004).

Given the numerous HIV risks associated with substance abuse as well as the high rates of substance abuse among male offenders, it is clear that substance abuse plays a central role in the overrepresentation of HIV/AIDS for men in criminal justice settings. However, few studies have explored the relationship between criminal justice involvement and specific HIV risk behaviors for substance abusing men, specifically male offenders in the community. It is critical to grasp the complexities of HIV risk behaviors for substance abusing male offenders in the community in order to inform the development of targeted and effective HIV prevention interventions for this vulnerable population. This paper examines the relationship between criminal justice involvement (arrest and incarceration) and drug and sexual HIV risk behaviors among a random sample of men in community-based methadone maintenance treatment programs (MMTPs). The study is designed to address two questions: Does recent criminal justice involvement increase the likelihood of HIV risk among men on methadone? Second, is a history of criminal justice involvement associated with increased prevalence of HIV for men on methadone?

\section{Methods \\ Participants}

A random sample was drawn from a population of 2,067 men enrolled in seven MMTPs in New York City. Of the 790 men who agreed to participate in a screening interview, 356 met eligibility criteria for the study. Eligibility criteria included men, 18 years or older, who were enrolled in an MMTP for at least 3 months, and who reported having a sexual relationship during the past year with a woman described as a girlfriend, spouse, regular sexual partner, or mother of participant's children. The criteria of having a female primary sexual partner was included due to the parent study's focus on HIV risk among heterosexual men. A total of 356 men completed a 90-min face-to-face, structured baseline interview administered by experienced male interviewers. Although subjects also participated in 6- and 12-month follow- 
up assessments, for the purposes of this study we used only the baseline data. A more detailed recruitment and sample description is available in a previous paper (El-Bassel et al. 2004).

\section{Measures}

Sociodemographic Characteristics-Self-reported, socio-demographic characteristics included age, race/ethnicity, education, monthly income and employment status in the past 6 months. Self-reported length of time in methadone maintenance treatment was also collected.

HIV Risk Indicators-Sexual HIV risk behaviors assessed in the 6 months prior to baseline assessment included unprotected vaginal or anal sex (with primary and other female partners), multiple female sexual partners, sex trading (buying or selling sex for drugs and/or money), and sex with someone known or suspected to be HIV positive and/or an injecting drug user (IDU). Baseline assessment also included obtaining histories of HIV tests and results of the most recent HIV test, and participants self-reported past diagnosis of sexually transmitted infections (STIs). Thorough substance use histories were collected, and substance use HIV risk was measured by injection drug use in the past 6 months and whether injection drug use equipment was shared in the past 6 months.

Criminal Justice Involvement-Participants were asked the following questions about their experience with police and the criminal justice system: "Have you ever been arrested (yes/no)? How many times in the past 6 months have you been arrested? Have you ever been incarcerated or in prison (yes/no)? In the past 6 months, have you been incarcerated or in prison (yes/no)?" In this paper, we use "recent arrest" and "recent incarceration" to refer to arrest or incarceration in the past 6 months. Lifetime arrest or incarceration refers to any arrest or incarceration over the participant's lifetime, including the past 6 months. Arrest and incarceration are treated as separate variables, as arrest is a less intense level of criminal justice involvement than incarceration.

\section{Data Analysis}

Descriptive statistics were generated for participant characteristics, HIV risk behavior, and criminal justice involvement. Sociodemographics and HIV risks were stratified by whether the participant had been arrested or incarcerated in the past 6 months (referred to as recent criminal justice involvement). Significant differences were identified between those with and without recent criminal justice involvement by using difference in means $t$-tests for continuous variables and Chi-square tests of independence for dichotomous variables. Logistic regression was used to estimate the association between participant HIV status and criminal justice history (both lifetime and past 6 month measures), with covariance adjustment for age, ethnicity, and education.

Second, we examined the association between recent arrest and recent incarceration and sexual and drug HIV risk behaviors. Because arrest is a less intense measure of criminal justice involvement than incarceration (i.e., not everyone arrested is also incarcerated) we treated each measure as an explanatory variable to examine differences in levels of recent criminal justice involvement. We first calculated unadjusted odds ratios for the bivariate associations between measures of criminal justice involvement and HIV risk by regressing each participant HIV risk behavior on each indicator of criminal justice involvement. Each participant HIV risk behavior was analyzed as an outcome variable with levels of criminal involvement as independent variables. Variables associated with recent criminal justice involvement were examined for use as controls in an adjusted model. The adjusted logistic regression models included one outcome variable and one measure of criminal justice involvement, with covariance adjustment for age, homelessness (past 6 months), time in methadone treatment, and whether the participant used any illicit drugs in the past 6 months. 


\section{Results}

\section{Criminal Justice Involvement}

A vast majority of the sample (94\%) reported at least one arrest in their lifetime, and 74\% indicated a lifetime history of incarceration or spending time in prison. In terms of recent criminal justice involvement, 92 men (26\%) reported being arrested in the past 6 months, and 39 men (11\%) reported being recently incarcerated or in prison. About one fourth $(26 \%)$ of the sample had been either arrested or incarcerated in the past 6 months.

\section{Sociodemographic Characteristics and Recent Criminal Justice Involvement}

Table 1 presents differences in HIV risks and background characteristics between men with and without criminal justice involvement in the past 6 months. No significant differences were found between these two groups in terms of current methadone dose, proportion of Latino and African American men, and recent levels of unemployment. However, significant differences emerged for several socio-demographic variables. Men with recent criminal justice involvement were about 3 years younger on average, slightly less educated, poorer, and averaged almost 2 years less in methadone treatment. Additionally, justice-involved men were more like to have recently been homeless (28\%) as compared to men with no recent criminal justice involvement $(13.3 \%$,). Furthermore, recent criminal justice involvement was associated with a higher likelihood of drug use in the past 6 months $(82.8 \%$ vs. $68.1 \%)$.

\section{HIV Risk Indicators}

From the total sample of 356 , about $14 \%(n=51)$ reported being HIV positive. Prevalence of HIV risk behaviors in the 6 months prior to assessment were reported as follows: Inconsistent condom use across all female partners: $n=243(68 \%)$, having more than one female sex partner: $n=86$ (24\%), having sex with a high risk partner: $n=58$ (16\%), injecting drugs: $n=86(24 \%)$, and sharing injection drug use equipment: $n=28(8 \%)$. In addition to primary and additional female partners, only four men reported having sex with men in the past 6 months.

Of the 33 men who reported trading sex for money or drugs in the past 6 months, 14 (42\%) reported buying sex, 4 (12\%) reported selling sex, and 15 (45\%) reported both buying and selling sex for money or drugs. Of the 188 men who had ever engaged in sex trading in their lifetime, $161(85 \%)$ did so only with women, $17(9 \%)$ mostly with women, $9(5 \%)$ with a balance of men and women, and only $1(<1 \%)$ engaged in sex trading with men only.

\section{HIV Risk Behavior and Recent Criminal Justice Involvement}

As can be seen in Table 1, men with recent criminal justice involvement were more likely to have unprotected sex, have more than one sex partner, and to have traded sex for money or drugs over the last 6 months. In addition, about $34 \%$ of the criminal justice involved group had recently injected drugs, while only $20.5 \%$ of the non-criminal justice involved group had engaged in this drug use HIV risk behavior. There were no significant differences between the two groups for HIV status, having sex with a risky partner (HIV positive status or IDU), sharing injection drug use equipment, or being diagnosed with a sexually transmitted infection.

\section{Association Between Criminal Justice Involvement and HIV Status}

Table 2 reports the logistic regression results (unadjusted and adjusted) of participant HIV status on past 6 months and lifetime measures of arrest and incarceration. Although lifetime arrest was not significantly associated with HIV status, a lifetime history of incarceration was strongly associated with the participant being HIV positive (adjusted $\mathrm{OR}=5.08, p<.01$ ). Neither recent incarceration nor recent arrest was significantly associated with HIV status. 


\section{Association Between Criminal Justice Involvement and Participant HIV Risks}

Table 3 presents a summary of logistic regression results (both adjusted and unadjusted) of participant's HIV risks on arrest and incarceration in the past 6 months. Arrest in the past 6 months was significantly associated with unprotected vaginal sex (adjusted $\mathrm{OR}=2.00, p<$. $01)$ and with an increased likelihood of multiple female sex partners in the past 6 months (adjusted $\mathrm{OR}=1.88, p<.05$ ). Neither recent arrest nor incarceration was significantly associated with unprotected anal sex with women or with sex with risky partners in the past 6 months.

Moreover, a strong association was found between recent criminal justice involvement and involvement in sex trading. Recent arrest was significantly associated with an increased likelihood of sex trading in the past 6 months (adjusted OR $=2.26, p<.05$ ), and recent incarceration demonstrated an even stronger relationship with sex trading activity (adjusted $\mathrm{OR}=3.40, p<.01$ ). To further specify the nature of sex trading activity, post hoc analyses separating the sex trading variable into buying sex for money or drugs and selling sex were conducted. Buying sex was associated with both recent arrest (adjusted $\mathrm{OR}=2.30, p<.05$ ) and incarceration (adjusted $\mathrm{OR}=2.86, p<.05$ ). Selling sex was associated with recent arrest (adjusted $\mathrm{OR}=2.85, p<.01$ ), and the strongest association was between selling sex and recent incarceration (adjusted OR $=5.69, p<.01$ ). The gender of partners with whom each sex trading act occurred was not specified in this study.

Lastly, criminal justice involvement in the past 6 months was associated with increased substance abuse HIV risk behaviors. Arrest and incarceration in the past 6 months were significantly associated with injecting drugs in the past 6 months (adjusted $\mathrm{OR}=1.95, p<$. 01 , adjusted $\mathrm{OR}=2.21, p<.05$ ). Sharing injection drug use equipment was not found to be significantly associated with either recent arrest or incarceration.

\section{Discussion}

The findings of this study are somewhat consistent with other studies examining the relationship between HIV status and criminal justice involvement. In this sample of men in MMTPs, participants with a lifetime history of incarceration were over five times more likely to report being HIV positive compared to participants with no history of incarceration, odds higher than those reported in previous studies (Maruschak 2005; Rich et al. 1999). Past research has often pointed toward the high rates of substance abuse to explain the levels of HIV/AIDS in prisons and jails (Harrison and Beck 2003; Swartz et al. 2004). Yet, in our sample comprised entirely of men with serious substance abuse histories, incarceration history indicated a significant increase in the likelihood of HIV positive status, suggesting that substance abuse alone may not explain the higher rates of HIV in justice-involved men. Men with both substance abuse problems and criminal justice involvement appear to share increased HIV risk over men with substance abuse alone.

A key finding in this study is the strong relationship between sex trading and recent criminal justice involvement. More specifically, criminal justice involvement was most closely associated with selling sex for money or drugs. Considering that only four men in the sample had sex with a man in the past 6 months, and the majority of those reporting sex trading over their lifetime reported it as primarily or exclusively with women suggests that the sex trading risks reported above entail primarily sexual activity with women. Nonetheless, the importance of this finding is magnified by the extremely high risk for HIV infection and transmission among male sex workers (Estcourt et al. 2000; Newman et al. 2004). Previous studies have linked sex trading behaviors to crack cocaine use (Joe and Simpson 1995; Latkin et al. 2003), which is consistent with current analysis of this study's data showing that those who used crack were more likely to be involved in sex trading (Orellana et al. 2007). 
Furthermore, our study finds that recent arrest (and not incarceration) is associated with increased likelihood of unprotected vaginal sex and multiple sex partners, which is consistent with prior research suggesting that criminal justice-involved men in the community have equivalent or even increased risk of HIV infection compared to their incarcerated counterparts (Belenko et al. 2004; Spaulding et al. 2002). These findings highlight the need for research on risky sex behaviors among justice-involved men in community settings, namely men recently arrested or briefly detained in jails and short-term correctional centers.

This study is limited by several factors. The sample focuses on men with female primary sexual partners, which may limit the generalizability of results to all men on methadone. Second, criminal justice involvement is measured in terms of arrest and incarceration, which are essentially consequences of illegal behavior. While it would be more difficult to measure actual criminal behavior in this sample, such measurement may serve to better explain related behaviors in terms of HIV risk. Lastly, this study bears the characteristics of a major metropolitan center in terms of criminal justice practices, substance abuse, and HIV/AIDS, which may not be generalizable to all communities. Continued research on the association between criminal justice involvement and HIV risk for men on methadone would benefit from studies representing a variety of geographic and population density areas.

Despite these limitations, this paper's findings on the associations between incarceration history and HIV status underscore the need for delivery of effective HIV prevention models for substance abusing men who have spent time in correctional settings. Engaging men with a history of incarceration in HIV prevention may reduce transmission among these men and their sexual partners, and methadone treatment programs could be an important venue for outreach. Although MMTPs have provided HIV education and counseling for over two decades, more attention needs to be given to gender-specific HIV prevention for men with a history of incarceration, which may constitute a large portion of the client population. Counselors and other staff could include a history of incarceration in their clinical assessment, and use this as a marker for HIV risk. Peer-led prevention interventions, a successful approach among IDUs (Latkin 1998), could target previously incarcerated men, which would move the focus beyond the individual level to social network and community norms (Metzger and Navaline 2003).

Additionally, those who are involved in both substance abuse and the criminal justice system have higher odds of selling sex for money or drugs. While the proportion of this sample that recently engaged in sex trading was relatively small, this group represents a population in great need of focused HIV prevention/education efforts. And although incarceration settings are identified as an opportunity for initiation of treatment and intervention (Rich et al. 1999), there is considerable need for prevention intervention efforts for justice-involved men in the community, where they have increased access to HIV risk opportunities.

The findings of this study emphasize the importance of future research that investigates the instrumental variables involved in the increased risk of HIV/AIDS for community-based, substance abusing offenders, as well as the need for focused prevention work with male offenders in the community. Although progress has been made in HIV prevention science, there are only a few evidence-based HIV intervention preventions for men with criminal justice involvement. More emphasis should be given to the design of gender-specific HIV intervention and dissemination of evidence-based models for such high-risk individuals in a variety of criminal justice settings. Addressing the severity of HIV risk for substance abusing men with criminal justice involvement will require integrated efforts at intervention and treatment from correctional settings, community criminal justice entities, substance abuse treatment, and the HIV/AIDS prevention community. 


\section{References}

Belenko S, Langley S, Crimmins S, Chaple M. HIV risk behaviors, knowledge, and prevention education among offenders under community supervision: A hidden risk group. AIDS Education and Prevention 2004;16:367-385. [PubMed: 15342338]

Booth RE, Watters JK, Chitwood DD. HIV risk-related sex behaviors among injection drug users, crack smokers, and injection drug users who smoke crack. American Journal of Public Health 1993;83(8): 1144-1148. [PubMed: 8342724]

Boutwell A, Rich JD. HIV infection behind bars. Clinical Infectious Diseases 2004;38:1761-1763. [PubMed: 15227624]

Braithwaite R, Stephens T. Use of protective barriers and unprotected sex among adult male prison inmates prior to incarceration. International Journal of STD \& AIDS 2005;16:224-226. [PubMed: 15829022]

Braithwaite RL, Arriola AR. Male prisoners and HIV prevention: A call for action ignored. American Journal of Public Health 2003;93:759-763. [PubMed: 12721138]

Braithwaite RL, Hammett T, Arriola KRJ. Introduction to the special issue: HIV/AIDS in correctional settings. AIDS Education and Prevention 2002;14(Suppl. B):1-6.

Bryant KJ. Expanding research on the role of alcohol consumption and related risks in the prevention and treatment of HIV/AIDS. Substance Use \& Misuse 2006;41(10):1465-1507. [PubMed: 17002990]

Campsmith ML, Nakashima AK, Jones JL. Association between crack cocaine use and high-risk sexual behaviors after HIV diagnosis. Journal of Acquired Immune Deficiency Syndromes 2000;25(2):192198. [PubMed: 11103051]

Chesney MA, Barrett DC, Stall R. Histories of substance use and risk behavior: Precursors to HIV seroconver-sion in homosexual men. American Journal of Public Health 1998;88(1):113-116. [PubMed: 9584015]

Colfax G, Guzman R. Club drugs and HIV infection: A review. HIV/AIDS 2006;42(10):1463-1469.

El-Bassel N, Gilbert L, Golder S, Wu E, Chang M, Fontdevila J. Deconstructing the relationship between intimate partner violence and sexual IPV risk among drug-involved men and their female partners. AIDS and Behavior 2004;8(4):429-439. [PubMed: 15690116]

Estcourt CS, Marks C, Rohrsheim R, Johnson AM, Donovan B, Mindel A. HIV, sexually transmitted infections, and risk behaviours in male commercial sex workers in Sydney. Sexually Transmitted Infections 2000;76:294-298. [PubMed: 11026887]

Freudenberg N, Daniels J, Crum M, Peridns T, Richie BE. Coming home from jail: The social and health consequences of community reentry for women, male adolescents, and their families and communities. American Journal of Public Health 2005;95(10):1725-1736. [PubMed: 16186451]

Glaze, LE. Probation and parole in the United States, 2001 (No NCJ 201135). Washington, DC: Bureau of Justice Statistics; 2003.

Hammett TM. HIV/AIDS and other infectious diseases among correctional inmates: Transmission, burden, and an appropriate response. American Journal of Public Health 2006;96:974-978. [PubMed: 16449578]

Hammett TM, Gaiter JL, Crawford C. Reaching seriously at-risk populations: Health interventions in criminal justice settings. Health Education \& Behavior 1998;25(1):99-120. [PubMed: 9474502]

Harrison, PM.; Beck, AJ. Prisoners in 2002. Bureau of Justice Statistics: Special Report, NCJ 200248. Washington, DC: Bureau of Justice Statistics; 2003.

Joe GW, Simpson DD. HIV risks, gender, and cocaine use among opiate users. Drug and Alcohol Dependence 1995;37:23-28. [PubMed: 7882870]

Krebs CP, Simmons M. Intraprison HIV transmission: An assessment of whether it occurs, how it occurs, and who is at risk. AIDS Education and Prevention 2002;14(Suppl. B):53-64. [PubMed: 12413193]

Latkin CA. Outreach in natural settings: The use of peer leaders for HIV prevention among injecting drug users' networks. Public Health Reports 1998;113(Suppl. 1):151-159. [PubMed: 9722820]

Latkin CA, Hua W, Forman VL. The relationship between social network characteristics and exchanging sex for drugs or money among drug users in Baltimore, MD, USA. International Journal of STD \& AIDS 2003;14:770-776. [PubMed: 14624742] 
Maruschak, LM. HIV in prisons and jails, 2002. Bureau of Justice Statistics Bulletin, NCJ 205333. Washington, DC: US Department of Justice; 2004. p. 1-11.

Maruschak, LM. HIV in Prisons, 2003. Washington, DC: U.S. Department of Justice, Office of Justice Programs; 2005.

Metzger DS, Navaline H. HIV prevention among injection drug users: The need for integrated models. Journal of Urban Health 2003;80(4):iii59-iii66. [PubMed: 14713672]

Molitor F, Truax SR, Ruiz JD, Sun RK. Association of methamphetamine use during sex with risky sexual behaviors and HIV infection among non-injection drug users. Western Journal of Medicine 1998;168 (2):93-97. [PubMed: 9499742]

Newman PA, Rhodes F, Weiss RE. Correlates of sex trading among drug-using men who have sex with men. American Journal of Public Health 2004;94:1998-2003. [PubMed: 15514243]

Orellana ER, El-Bassel N, Gilbert L, Epperson M. Sex trading and HIV risk among men in methadone maintenance treatment. 2007 in press.

Pollack H, Khoshnood K, Altice F. Health care delivery strategies for criminal offenders. Journal of Health Care Finance 1999;26(1):63-77. [PubMed: 10497752]

New York City Department of Health. Report on HIV Seroprevalence in New York City in 1998. New York: New York City Department of Health; 1999.

Rich JD, Dickinson BP, Macalino G, Flanigan TP, Towe CW, Spaulding A. Prevalence and incidence of HIV among incarcerated and reincarcerated women in Rhode Island. Journal of Acquired Immune Deficiency Syndromes 1999;22:161. [PubMed: 10843530]

Seal DW. HIV-related issues and concerns for imprisoned persons throughout the world. Current Opinion in Psychiatry 2005;18:530-535. [PubMed: 16639113]

Spaulding A, Stephenson B, Mecalino G, Ruby W, Clarke JG, Flanigan TP. Human immunodeficiency virus in correctional facilities: A Review. HIV/AIDS 2002;35:305-312.

Springer SA, Altice FL. Managing HIV/AIDS in correctional settings. Current HIV/AIDS Reports 2005;2 (4):165-170. [PubMed: 16343373]

Stephens T, Braithwaite R, Tiggs C. Correlates of inmates' self-reported HIV/AIDS risk behaviors, prior incarceration, and marijuana use. The American Journal of Drug and Alcohol Abuse 2004;30:287298. [PubMed: 15230077]

Stephens TT, Braithwaite R, Sprauve NE, Louis TR. Predictors of prior incarceration and alcohol use among soon-to-be-released adult male inmates. Journal of Correctional Health Care 2006;12:4-11.

Swartz JA, Lurigio AJ, Weiner DA. Correlates of HIV-risk behaviors among prison inmates: Implications for tailored AIDS prevention programming. The Prison Journal 2004;84:486-504.

Vlahov D, Putnam S. From corrections to communities as an HIV priority. Journal of Urban Health: Bulletin of the New York Academy of Medicine 2006;83:339-348. [PubMed: 16739034]

Wohl AR, Johnson DF, Lu S, Jordan W, Beall G, Currier J. HIV risk behaviors among African American men in Los Angeles County who self-identify as heterosexual. Journal of Acquired Immune Deficiency Syndromes 2002;31:354-360. [PubMed: 12439213] 


\section{Table 2}

Logistic regression results of participant's HIV status on lifetime and recent arrest and incarceration: unadjusted and adjusted odds ratios and $95 \%$ confidence intervals

\begin{tabular}{|c|c|c|c|c|}
\hline \multirow[t]{3}{*}{ Independent variable } & \multicolumn{4}{|c|}{ Dependent variable } \\
\hline & \multicolumn{4}{|c|}{ Participant is HIV positive } \\
\hline & Unadjusted & $95 \% \mathrm{CI}$ & Adjusted $^{a}$ & $95 \% \mathrm{CI}$ \\
\hline Participant was ever arrested & 1.72 & $(.4,7.6)$ & 1.69 & $(.4,7.6)$ \\
\hline Participant was ever incarcerated or in prison & $4.76^{* *}$ & $(1.7,13.6)$ & $5.08^{* *}$ & $(1.7,14.8)$ \\
\hline Participant was arrested in past 6 months & 0.49 & $(.2,1.1)$ & 0.59 & $(.3,1.3)$ \\
\hline Participant incarcerated in past 6 months & 0.47 & $(.1,1.6)$ & 0.52 & $(.2,1.8)$ \\
\hline${ }^{*} p<.01$ & & & & \\
\hline
\end{tabular}


Table 3

Summary of logistic regression results of participant HIV risks on arrest and incarceration: unadjusted and adjusted odds ratios and $95 \%$ confidence intervals

\begin{tabular}{|c|c|c|c|c|}
\hline \multirow[t]{3}{*}{ Dependent variable } & \multicolumn{4}{|c|}{ Independent variable } \\
\hline & \multicolumn{2}{|c|}{ Arrested in the past 6 months } & \multicolumn{2}{|c|}{ Incarcerated in the past 6 months } \\
\hline & Unadjusted & Adjusted $^{b}$ & Unadjusted & Adjusted $^{b}$ \\
\hline Unprotected vaginal sex in the past 6 months & $2.12^{* *}(1.22,3.70)$ & $2.00^{* *}(1.11,3.54)$ & $1.50(.70,3.19)$ & $1.46(.67,3.19)$ \\
\hline $\begin{array}{l}\text { Unprotected anal sex in the past } 6 \text { months (female } \\
\text { partner) }\end{array}$ & $1.67(.94,2.95)$ & $1.37(.75,2.52)$ & $1.13(.49,2.58)$ & $.91(.38,2.18)$ \\
\hline More than one female sexual partner in the past 6 months & $1.93^{* *}(1.14,3.26)$ & $1.88^{*}(1.09,3.24)$ & $1.91(0.94,3.86)$ & $1.81(.88,3.73)$ \\
\hline Engaged in sex trading in the past 6 months & $2.31^{*}(1.11,4.83)$ & $2.26^{*}(1.04,4.90)$ & $3.66^{* *}(1.56,8.6)$ & $3.40^{* *}(1.41,8.20)$ \\
\hline Bought sex for money or drugs in past 6 months & $2.18(1.0,4.76)$ & $2.30 *(1.00,5.27)$ & $2.93^{*}(1.16,7.40)$ & $2.86^{*}(1.09,7.49)$ \\
\hline Sold sex for money or drugs in past 6 months & $3.45^{* *}(1.36,8.80)$ & $2.85^{* *}(1.07,7.63)$ & $7.18^{* *}(2.7,19.2)$ & $5.69^{* *}(2.05,15.8)$ \\
\hline Sex with someone HIV + or IDU in the past 6 months & $1.50(0.82,2.76)$ & $1.38(.73,2.63)$ & $2.26^{*}(1.05,4.8)$ & $1.95(.88,4.30)$ \\
\hline Injected drugs in the past 6 months ${ }^{a}$ & $2.07^{* *}(1.23,3.50)$ & $1.95^{* *}(1.13,3.37)$ & $2.45(1.23,4.90)$ & $2.21^{*}(1.08,4.49)$ \\
\hline Shared IDU equipment in the past 6 months ${ }^{a}$ & $1.97(0.89,4.39)$ & $1.83(.77,4.36)$ & $2.44(0.92,6.44)$ & $1.88(.66,5.37)$ \\
\hline $\begin{array}{l}* x<.05, \\
* * \\
p<.01\end{array}$ & & & & \\
\hline
\end{tabular}

